\title{
Screening of differentially expressed gene in HIV/HCV co-infected patients
}

\author{
zhengrong Yang ${ }^{1 *}$, Jing Zhao ${ }^{1}$, Lin Chen ${ }^{1}$, Jin-Quan Cheng ${ }^{1}$, Ming-Liang $\mathrm{He}^{2}$ \\ From $16^{\text {th }}$ International Symposium on HIV and Emerging Infectious Diseases \\ Marseille, France. 24-26 March 2010
}

\section{Background}

Most research has shown that HIV/HCV coinfected people tend to experience faster liver disease progression and develop more AIDS-defining illnesses than mono infected patients. The objective of this study was to screen the differentially expressed genes in $\mathrm{HIV} / \mathrm{HCV}$ co-infected patients, and further investigate its possible mechanism.

\section{Methods}

Eligibility Criteria including 20-40 year-old male, similar social-economic background, treatment naive, same transmission route methods (Sex or Injection drug), and CD4 count $>200$ cells $/ \mathrm{mm}^{3}$. All research objects signed on informed consent. The project proceeded after approval of the local REC.

Human blood samples were collected from 13 HIV/ HCV coinfected patients, 8 mono infected patients, and 8 normal control. At first, CD4 and CD8 positive T cells were separated with magnetic activated cell sorting, then followed by RNA extraction. After reverse transcripted to cDNA with anchor primer and amplified with random primers, the products were run on PAGE electrophoresis followed by silver stain. The bands with different expression level were extracted from gel and cloned for sequencing. The expression changes were confirmed by SYBR Green real-time quantitative PCR.

\section{Results}

Totally 307 differentially expressed bands were extracted. 25 fragments were sequenced and found 3 for ring-finger protein 141(RNF141), 5 for cyclic AMP phosphoprotein (ARPP-19), 5 for mitochondrial cytochrome (cytochrome c and b-245). Most of the other fragments were function unkown sequences located on
$\mathrm{X}$ chromosome and mitochondrial. The change of expression of cytochrome $\mathrm{c}$ and ARPP-19 were confirmed with a significant down-regulation in $\mathrm{HIV} / \mathrm{HCV}$ coinfected patients and a slight down-regulation in mono-infected group compared to negative population.

\section{Discussion}

1. The expression of RNF-141, ARPP-19, cytochrome c and b-245 mRNA are probably associated with HIV/ HCV co-infection.

2. The technique of mRNA differential display together with SYBR Green quantitative real-time PCR is useful for screening for differentially expressed genes in HIV/HCV co-infection.

3. Since mitochondrial cytochrome has been reported to associated with prognosis of AIDS. Is it possible to act as a useful marker for prognosis of $\mathrm{HIV} / \mathrm{HCV}$ coinfection? More clinical data are needed.

\section{Author details}

${ }^{1}$ Shenzhen Center for Disease Control and Prevention, Shenzhen, PR China. ${ }^{2}$ School of Public Health and Primary Care, The Chinese University of Hong Kong, Hong Kong, SAR, PR China.

Published: 11 May 2010

doi:10.1186/1742-4690-7-S1-O3

Cite this article as: Yang et al:: Screening of differentially expressed gene in HIV/HCV co-infected patients. Retrovirology 2010 7(Suppl 1):O3.

* Correspondence: yangzr1226@yahoo.com.cn

'Shenzhen Center for Disease Control and Prevention, Shenzhen, PR China 